\title{
AN IDEAL CLASS INVARIANT OF KNOTS
}

\author{
R. H. FOX AND N. SMYTHE
}

In this note the row and column ideal class invariants of a matrix (cf. [3]) are applied to the Alexander matrix of a knot to give an invariant for knots. We give an example of the use of this invariant to distinguish a pair of knots which cannot be distinguished by their elementary ideals, torsion numbers, or linking invariants. ${ }^{1}$

1. Let $M$ be an $m \times n$ matrix over a commutative integral domain $R$. For any integer $k$ consider the $k$ th compound matrix $M^{(k)}$, the

$$
\left(\begin{array}{l}
m \\
k
\end{array}\right) \times\left(\begin{array}{l}
n \\
k
\end{array}\right)
$$

matrix whose entries are the $k \times k$ minor determinants of $M$, rows and columns written in lexicographic order. If the rank of $M$ is $r$, $M^{(k)}=0$ for $k$ greater than $r$, whereas $M^{(r)}$ contains some nonzero element. Further, $M$ is equivalent to a diagonal matrix $N$ over the quotient field of $R$ and, as may be easily seen, $M^{(k)}$ is equivalent to $N^{(k)}$. Since the rank of $N^{(r)}$ is 1 , the rank of $M^{(r)}$ is 1 .

We recall that two ideals $I, J$ are said to be equivalent if there exist nonzero principal ideals $P, Q$ such that $P I=Q J$. The ideal classes of a ring under this relation form a semi-group with the principal ideal class as identity.

By the $i$ th row ideal $\rho_{i}$ of $M$ is meant the ideal generated by the elements of the $i$ th row of $M^{(r)}$. Suppose that both the $i$ th and $k$ th rows contain nonzero elements. Writing $M^{(r)}=\left\|m_{i j}\right\|$ and choosing $q$ such that $m_{i q}$ is nonzero, we have for any $j$

$$
\operatorname{det}\left\|\begin{array}{ll}
m_{i q} & m_{i j} \\
m_{k q} & m_{k j}
\end{array}\right\|=0
$$

since $M^{(r)}$ is of rank 1. Thus $m_{i q} m_{k j}=m_{k q} m_{i j}$ and $\left(m_{i q}\right) \rho_{k}=\left(m_{k q}\right) \rho_{i}$, where $(z)$ denotes the principal ideal generated by $z$. It follows that $m_{k q}$ is nonzero and thus that any two nonzero row ideals are equivalent. This equivalence class of ideals is called the row class of $M$. Similarly we may define the column class of $M$.

2. There is defined for matrices over a ring $R$ an equivalence relation by means of the following operations (see $[1$, p. 101]):

Received by the editors May 10, 1963.

1 We are indebted to $\mathrm{P}$. $\mathrm{H}$. Monsky for his suggestions concerning the algebra of the example. 
(i) Permuting rows and columns;

(ii) Adjoining a row of zeros;

(iii) Adding to some row a linear combination of other rows;

(iv) Adding to some column a linear combination of other columns;

(v) Adjoining a new row and column each of whose elements is zero except for the entry 1 in their intersection.

Let $G$ be the group of a knot, $H$ its abelianization, $J H$ the integer group ring of $H$, i.e., the ring of $L$-polynomials. Further let $A(t)$ be an Alexander matrix for $G, \Delta(t)$ the Alexander polynomial of the knot, and $E$ an ideal in $J H$ generated by one of the irreducible factors of $\Delta(t)$. Then $A(t)$ is a singular matrix over the integral domain $J H / E$. The Tietze operations on the presentation of the group $G$ result in transformations of $A(t)$ by means of the above operations over the ring $J H$, and thus over the ring $J H / E$.

Now the row and column class of a matrix remain invariant under the operations (i)-(v). This is clear for (i)-(iv); the operation (v) increases the rank of the matrix, but does not change the elements of the row ideals (column ideals) of the matrix. It follows that the row class and column class of the Alexander matrix over $J H / E$ are invariants of the knot.

3. The ring $J H / E$ being somewhat unfamiliar, it is useful to give a few elementary facts about it before going on to the example. Suppose $w$ is a root of the irreducible factor $a(t)$ of $\Delta(t)$. If $P(t), Q(t)$ are elements of $J H$ such that $P(w)=Q(w), P(t)-Q(t)$ has $w$ for a root. Then every root of $a(t)$ is a root of $P(t)-Q(t)$ and therefore $P(t)-Q(t) \equiv 0 \bmod a(t)$. Thus $P(t) \equiv Q(t) \bmod E$ if and only if $P(w)$ $=Q(w)$. JH/E may therefore be imbedded in the algebraic number field $k(w)$, where $k$ is the field of rationals. The norm of $P(w)$ in $k(w)$ serves as a norm for $P(t)$.

Since $\Delta(t)$ may be written in the form $1+c_{1}(t-1)+\cdots$ $+c_{2 h}(t-1)^{2 h}$, we note that $t-1$ is a unit in $J H / E$.

For a knot of genus 1 (see [2]) an Alexander matrix is

$$
A(t)=\left\|\begin{array}{lrl}
1+v_{12}(t-1) & -v_{11}(t-1) & 0 \\
v_{22}(t-1) & 1-v_{21}(t-1) & 0
\end{array}\right\|
$$

and the Alexander polynomial $\Delta(t)=\gamma t^{2}+(1-2 \gamma) t+\gamma$, where $\gamma=v_{11} v_{22}-v_{12} v_{21}$. Since $\gamma(1-t)^{2} \equiv-t \bmod \Delta(t)$, every divisor of $\gamma$ is a unit in $J H / E$. If $P(t)$ is in $J H$, then $P(w)$ is an algebraic integer of $k(w)=k(\sqrt{ }(1-4 \gamma))$ divided by some power of $\gamma$, and its norm is a rational number with denominator some power of $\gamma$. It is not difficult 
to see that $P(t)$ is a unit in $J H / E$ if and only if the numerator of its norm is a product of divisors of $\gamma$.

Since $A(t)$ is of rank 1 in $J H / E$, the row class of $A(t)$ is represented by the ideal $\left(1+v_{12}(t-1),-v_{11}(t-1)\right)$, i.e., $\left(1+v_{12}(t-1), v_{11}\right)$.

4. We compare two knots with $\gamma=53 . k_{1}$ is any knot with $v_{11}=5$, $v_{22}=11, v_{12}=2, v_{21}=1 . k_{2}$ is any knot with $v_{11}=1, v_{22}=53, v_{12}=1$, $v_{21}=0$. These knots have the same elementary ideals, torsion numbers, linking invariants and Minkowski units. As the referee remarks, $k_{1}$ may be taken to be the pretzel $\operatorname{knot}(25,-3,13)$, and $k_{2}$ the pretzel knot $(107,-1,3)$, in which case by Trotter [4] $k_{1}$ is noninvertible and $k_{2}$ is invertible.

The row class of the knot $k_{2}$ is clearly principal.

If $R$ is the ring of integers of $k(\sqrt{ }(-211))$, it is readily seen that $J H / \Delta(t)$ may be imbedded in $k(\sqrt{ }(-211))$ as the ring $R^{\prime}=R[1 / 53]$. Under this isomorphism the ideal $(5,2 t-1)$, which represents the row class of $k_{1}$, becomes the ideal $I=(5,(52+\sqrt{ }(-211)) / 53)$. Consider the ideal $J=(5,52+\sqrt{ }(-211))$ in $R$. Then $J R^{\prime}=I$. If $I$ is principal, generated by $\alpha, J R^{\prime}=\alpha R^{\prime}$. We may assume $\alpha \in J$ by replacing $\alpha$ by $\alpha \cdot 53^{n}$ if necessary, so that $J \supset \alpha R$. Noting that the ring of integers of $k(\sqrt{ }(-211))$ is a Dedekind domain, we let $K=\alpha J^{-1}$. Then $K \subset R$ and $K R^{\prime}=\alpha J^{-1} R^{\prime}=R^{\prime}$. It follows that $K$ is a product of prime ideals dividing $53 R$. But the ideal $53 R$ is a product of the two principal ideals $\left(\frac{1}{2}(1+\sqrt{ }(-211))\right)$ and $\left(\frac{1}{2}(1-\sqrt{ }(-211))\right)$. Since the norm of these ideals is 53 they are prime ideals. Thus if $I$ were principal in $R^{\prime}, K$ would be principal in $R$ and therefore $J$ principal in $R$.

But $1 \notin J$ since every element of $J$ has norm divisible by 5.5 is a prime in $R$ since $4 \cdot 5=a^{2}+211 b^{2}$ has no solution in integers, so that if $J$ were principal it would be generated by 5 . On the other hand $52+\sqrt{ }(-211)$ is not a multiple of 5 . Thus the row class of $k_{1}$ is not principal, distinguishing it from $k_{2}$.

\section{REFERENCES}

1. R. H. Crowell and R. H. Fox, Introduction to knot theory, Ginn, Boston, 1963.

2. R. H. Fox, Homology characters of the cyclic coverings of the knots of genus one, Ann. of Math. 71 (1960), 187-196.

3. E. Steinitz, Rechteckige Systeme und Moduln in algebraischen Zahlkörpern. I, Math. Ann. 71 (1912), 328-354.

4. H. F. Trotter, Noninvertible knots exist, Topology 2 (1964), 245-280.

Princeton UNIVERSITY 